\title{
Fusion in a Staged Z-pinch
}

\author{
H.U. Rahman $\cdot$ P. Ney $\cdot$ N. Rostoker $\cdot$ F.J. Wessel
}

Received: 19 May 2008 / Accepted: 16 March 2009 / Published online: 26 March 2009

(C) The Author(s) 2009. This article is published with open access at Springerlink.com

\begin{abstract}
A Staged Z-pinch (H.U. Rahman, F.J. Wessel, N. Rostoker, Phys. Rev. Lett. 74:714, 1995), configured for a $100 \mathrm{~ns}, 2 \mathrm{MJ}$ implosion accelerator, is studied using the 2$1 / 2 \mathrm{D}$, radiation-MHD code, MACH2. The Z-pinch is configured as a cylindrical, high-atomic number plasma shell that implodes radially onto a co-axial, plasma target, for example: Xenon onto a 50:50 mixture of Deuterium-Tritium. During implosion a shock develops in the plasma liner, producing a conduction channel at the Xe/DT interface as the mass Xe accumulates, and preheating the DT target. During subsequent acceleration and compression the Xe/DT interface remains stable, even as the outer surface of the Xe shell develops RT instabilities. At peak implosion the simulated fusion-energy yield is 7.6 MJ, producing an energy gain of 3.8 .
\end{abstract}

Keywords Magneto-inertial fusion

The $\mathrm{Z}$ pinch has been studied as a means to attain highenergy-density plasma compressions for many decades (Teller 1981). During implosion the Z-pinch becomes Rayleigh-Taylor unstable; as a light fluid (pinch-magnetic field) pushes against a heavy fluid (plasma). In the linearregime of analytic treatment, plasma perturbations grow as

H.U. Rahman · N. Rostoker · F.J. Wessel ( $\varangle)$

Physics and Astronomy, Univ. of California, Irvine, CA 92697,

USA

e-mail: frank.wessel@uci.edu

P. Ney

Dept. Physics, Mount San Jacinto College, Menifee, CA 92584, USA
(Chandrasekar 1981),

$\xi=\xi_{0} e^{\gamma t}$

where $\xi_{0}$ is the initial perturbation, $\gamma=\sqrt{g k}$ is the growth rate, $g$ is the acceleration, $k$ is the wavenumber, and $t$ is the time. A rough approximation for the distance over which the plasma is accelerated is the initial radius, and using, $R_{0}=$ $g t^{2} / 2, \xi$ is re-written as,

$\xi=\xi_{0} e^{\sqrt{2 R_{0} k}}$.

Hence, for a given mode number the RT instability grows exponentially with initial radius, but is independent of the magnitude of the acceleration.

The pinch energy is approximately equal to the work done on the pinch,

$W=\int \vec{F} \cdot d \vec{r}=\frac{I_{\max }^{2} h}{c^{2}} \ln \left(\frac{R_{0}}{R_{f}}\right)$,

where $I$ is the maximum current, $h$ is the pinch height, and $R_{f}$ is the final radius of the pinch. Consideration of these tradeoffs suggests that the initial pinch radius should not be so large that instability arises, nor so small as to limit energy accumulation.

The Staged Z-pinch (Rahman et al. 1995) is a specific Z-pinch configuration comprised of a high-atomic number (Xenon) liner imploding onto a coaxial-plasma target (50:50 mixture of Deuterium-Tritium). Configured for a $2 \mathrm{MJ}$, sinusoidal, $110 \mathrm{~ns}$ (quarter period), $17 \mathrm{MA}$ implosion accelerator (similar to the parameters of the Sandia $\mathrm{Z}$ Facility) the configuration is modeled using the singlefluid, magneto-hydrodynamic, two-and-a-half dimensional (2-1/2D), time-dependent, MACH2 code; the equations of which are described elsewhere (Peterkin et al. 1998; Ney 


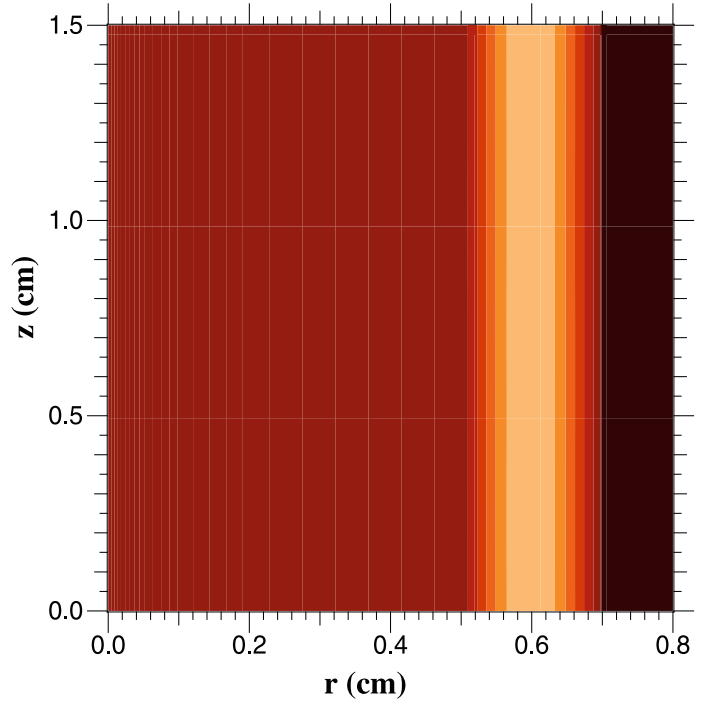

Fig. 1 Staged Z-pinch: $1.5-\mathrm{cm}$ high, 0.7-cm radius, 0.2-cm thick Xe liner, with an uniform, 50:50 coaxial fill of DT

et al. 2001). MACH2 treats separately the electron, ion and radiation temperatures and calculates resistive and thermal diffusion using established transport models. MACH2 calculates flux-limited, single-group, implicit-radiation diffusion. The plasma equation-of-state is determined from SESAME look-up tables. The generalized Ohm's Law includes the Hall Effect and thermal-source terms for B fields.

The pinch is modeled as a $1.5-\mathrm{cm}$ high, right-circular cylinder, as shown in Fig. 1. The outer radius of the axiallyuniform, radial-Gaussian density, 0.2-cm thick, Xe liner is $0.7-\mathrm{cm} ; M_{X e}=3.59 \times 10^{-2} \mathrm{gm}$. DT fills the liner uniformly to $0.5-\mathrm{cm}$ radius; $M_{D T}=3.46 \times 10^{-4} \mathrm{gm}$. The discharge electrodes form the top and bottom boundaries of the simulation and are perfectly conducting. The simulated volume is resolved into 320 radial and 64 axial cells. The calculation is axially Eulerian and radially Lagrangian; the radial motion of the computational grid is tied to the plasma pressure gradient. Instability wavelengths as small as $0.2 \mathrm{~mm}$ are modeled. The (cold-start) temperature is $2 \mathrm{eV}$ and a $10^{-2}$ random perturbation is applied throughout the volume.

The discharge current initiates on the outer surface of the Xe liner. A $\vec{J} \times \vec{B}$ force accelerates the Xe liner inward. The high-atomic-number of $\mathrm{Xe}$ enhances radiation losses, allowing the plasma liner to remain cool and resistive during implosion.

Figure 2 is a simulated, R-Z, iso-contour profile of the axial-current density, $94 \mathrm{~ns}$ into the implosion. At this time the pinch radius has decreased to $0.28 \mathrm{~cm}$ and there are early indications of the Rayleigh-Taylor instability on the outer surface of the Xe liner (wavelength: $\lambda \sim 1.0 \mathrm{~mm}$ ). Note that the Xe/DT interface boundary remains uniform and stable.

An axial average of the Fig. 2 data produces the radial profile shown in Fig. 3, indicating that the largest current

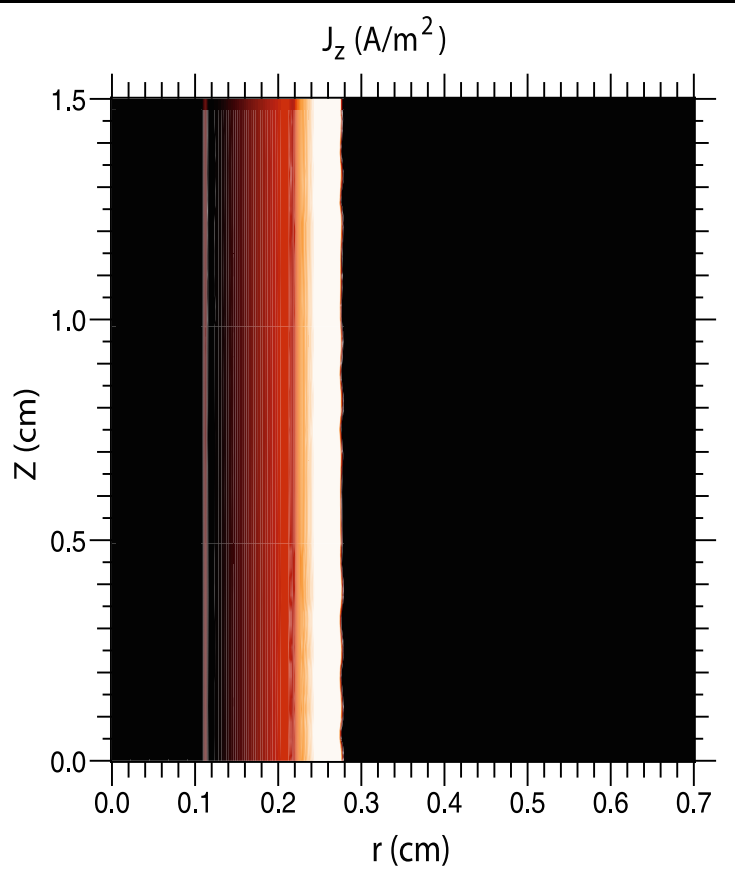

Fig. 2 Axial-current density "iso-surfaces," at 94 ns

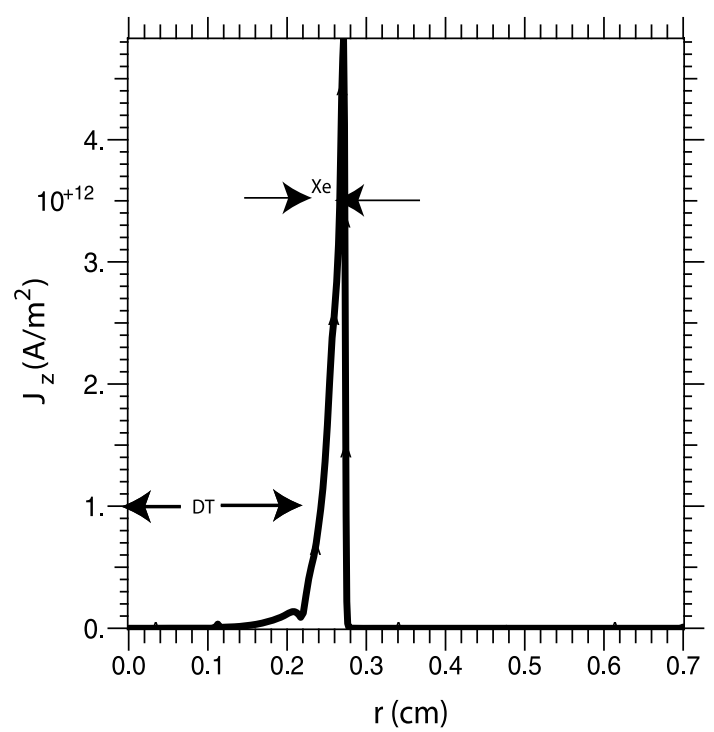

Fig. 3 Axially-averaged current density, at 94 ns

density exists in the Xe liner. A smaller component of diffused current extends into the DT. The level of diffused current at the interface continues to build during the implosion, and near the peak implosion is compressed, and amplified, by the Xe liner inertia. Flux compression by this mechanism leads to the generation of an ultra-high magnetic field, confined between the Xe and DT (Rahman et al. 1995).

Figure 4 displays the axially-averaged, logarithmicelectron density at four time steps leading up to maximum compression: 107, 108, 109, and at 109.41 ns (note the radial scale). The electron density, $n_{e}$, is highest in the Xe 


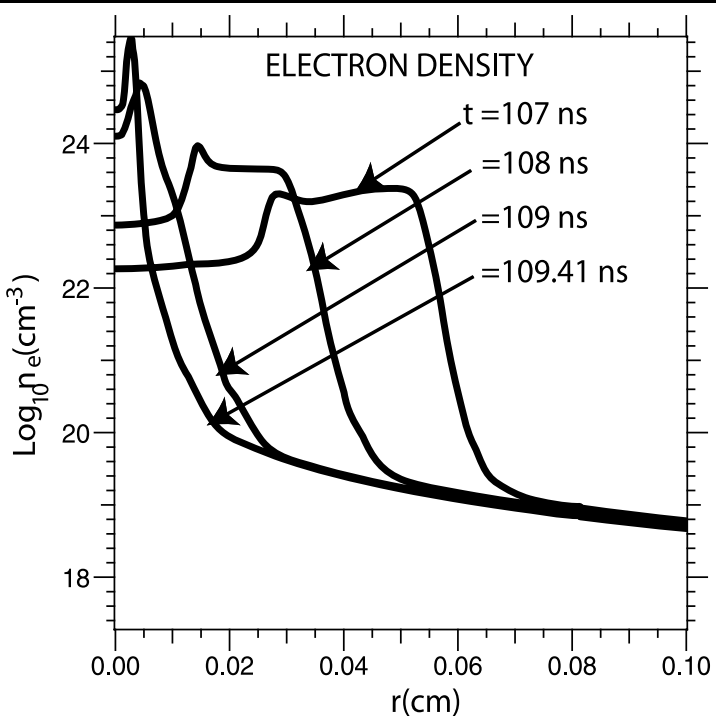

Fig. 4 Axially-averaged logarithmic-electron density at 107, 108, 109 and $109.41 \mathrm{~ns}$

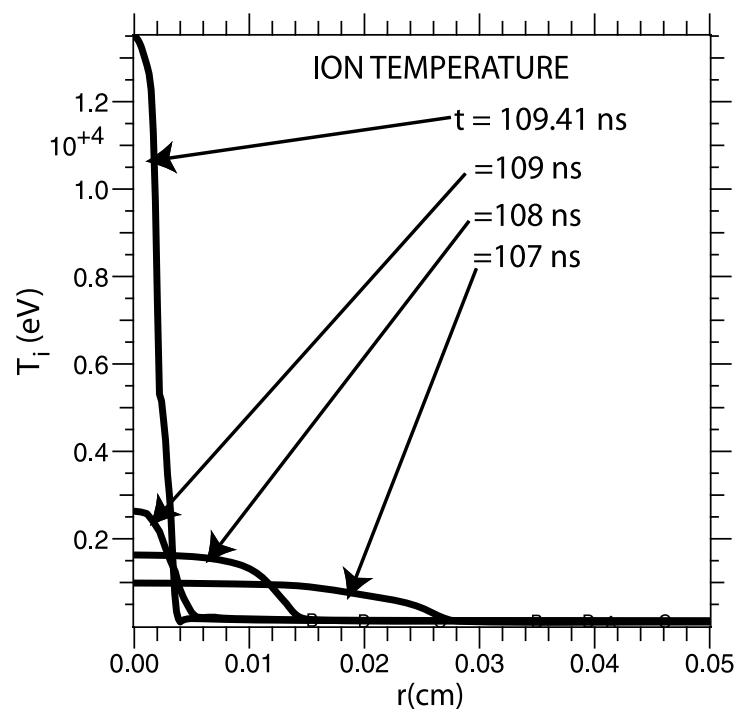

Fig. 5 Axially-averaged ion-temperature at 107, 108, 109 and 109.41 ns

liner. The peak value is, $n_{e}(\mathrm{Xe})=5 \times 10^{25} \mathrm{~cm}^{-3}$, for Xe and approximately a factor of 10 less for DT, $n_{e}(D T)=$ $n_{i}(D T)=5 \times 10^{24} \mathrm{~cm}^{-3}$. A high-charge-state, tenuous Xe plasma tail is present at larger radius, that trails behind the implosion front.

The ion-temperature profiles, corresponding to the same time steps, are shown in Fig. 5 (note the radial scale). The ion temperature is highest in the DT and lowest in the Xe. At peak compression the DT ion temperature attains a value, $T_{i}(D T)=13.5 \mathrm{keV}$, whereas the $\mathrm{Xe}$ ion temperature remains below $0.1 \mathrm{keV}$.

Inspection of the 2-D source data for the axially-averaged, time-stepped profiles of Figs. 4 and 5 reveal that shocks

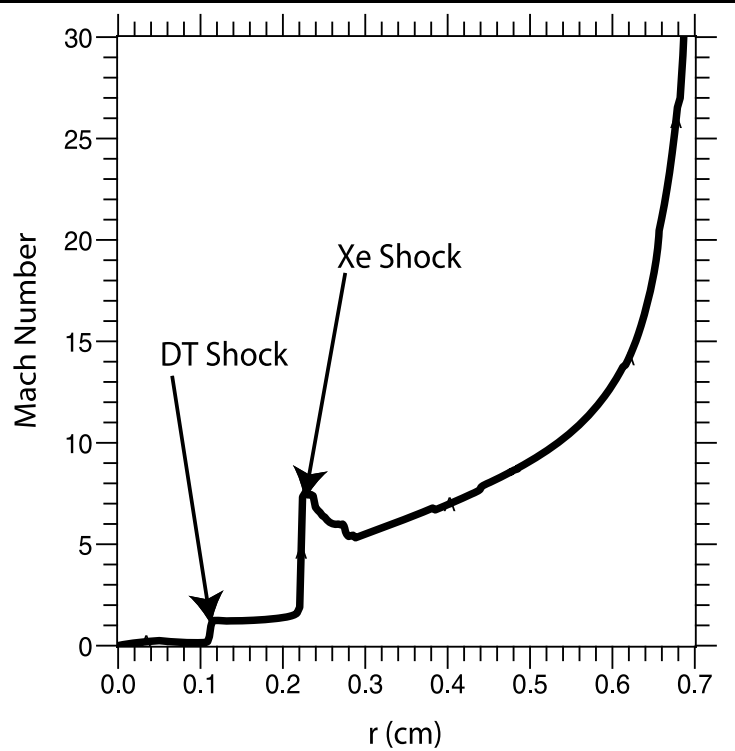

Fig. 6 Axially-averaged Mach number, at 94 ns

form early and continue until peak implosion, oscillating within the DT and reflecting at the interface. The Mach number, $M=V_{r} / C_{s}$, computed at $94 \mathrm{~ns}$ is plotted in Fig. 6 . Shock fronts are evident at $0.22-\mathrm{cm}$ radius, in the $\mathrm{Xe}$, and at $0.12 \mathrm{~cm}$ radius, in the DT. Oscillating shocks (pre)heat the DT, adding internal energy to the plasma that would otherwise be limited by adiabatic compression alone.

In a liner-on-target compression an under appreciated effect of shocks is their ability to stabilize the plasma (Rostoker and Tahsiri 1978). This enhancement, and the specific timing that occurs naturally in the Staged Z-pinch, is critical for the efficient production of fusion energy. Shock stabilization, observed previously using a ns pulsed laser system (Rahman et al. 2004), was thought to be due to the compression of a low-mass-density target by a high-mass-density liner.

Shown in Fig. 7 is an R-Z, iso-contour profile of the ion density at $109 \mathrm{~ns}$, just before peak compression (note the radial scale). At this time the Xe/DT interface has become RT unstable, the amplitude of which extends beyond the liner to a radius, $r>0.02 \mathrm{~cm}$, into regions of high-charge state. The time at which this penetration occurs facilitates the formation of hot-spots in the DT, which are visible on-axis.

The (combined) Lawson Product, for the peak density and temperature given above, and a nominal confinement time of, $\tau \sim 0.2 \mathrm{~ns}$, is, $n T \tau \sim 1.3 \times 10^{19} \mathrm{~cm}^{-3} \mathrm{keV} \mathrm{s}^{-1}$, which is adequate for fusion. Figure 8 characterizes the energy components and yield, over the time interval 100$110.5 \mathrm{~ns}$.

Fusion burn begins abruptly at $109.2 \mathrm{~ns}$, just after a rapid decrease in the plasma kinetic energy and a corresponding increase in the ion energy. The total fusion yield is approximately $8 \mathrm{MJ}$, which is 4 times the stored capacitor-bank 


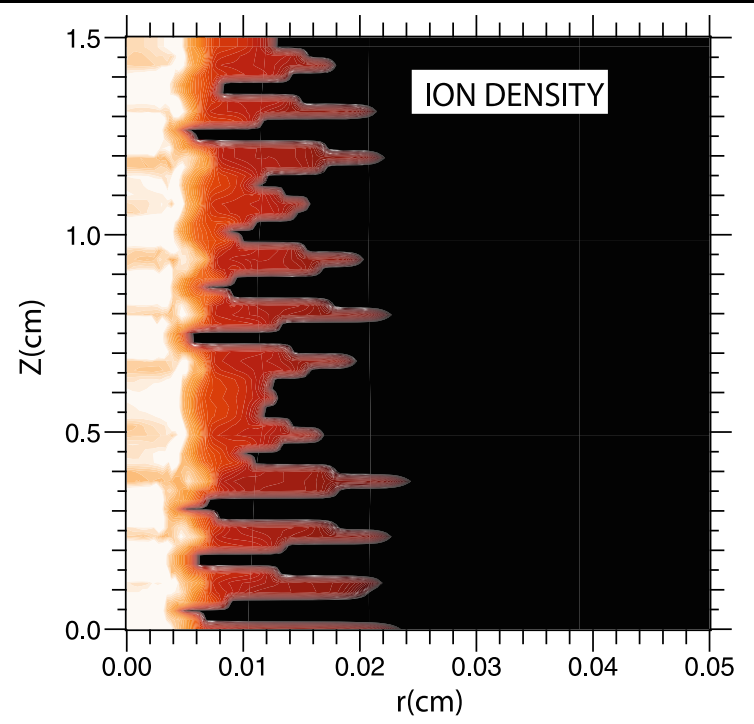

Fig. 7 Ion density "iso-surface" at $109 \mathrm{~ns}$; the ion temperature iso-surface at this time index is similar

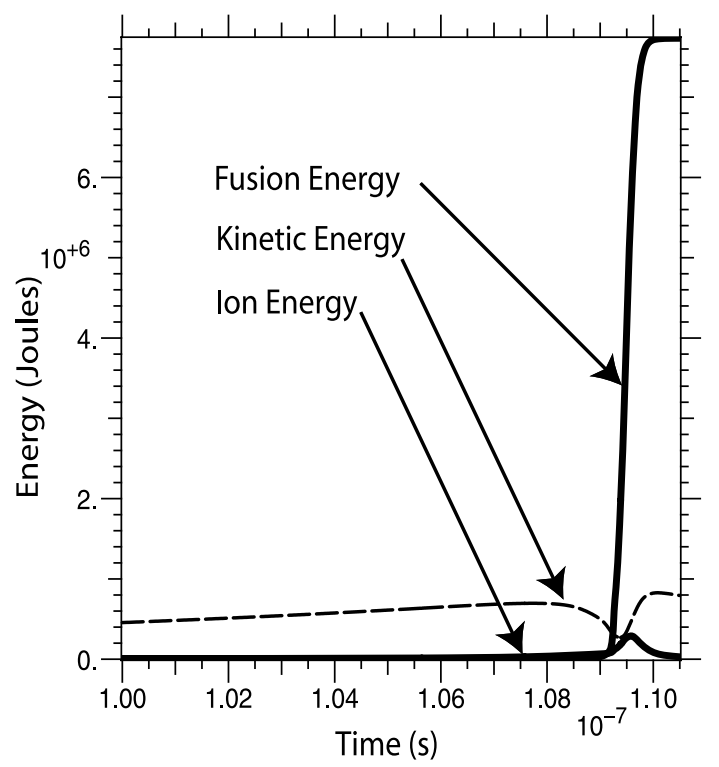

Fig. 8 Fusion energy, circuit energy, kinetic energy, and ion energy vs. time

energy. The corresponding thermonuclear-neutron yield is $2.76 \times 10^{18}$.

The onset of fusion increases the internal energy of the DT, causing it to expand radially outward as it compresses the Xe liner to high density. At 110.5 ns the pinch becomes grossly unstable to the RT instability (light-fluid targetplasma pushing against the heavy-fluid Xe liner) disrupting the circuit current and halting the calculation.

Figure 9 compares the total-radiated energy, near peak compression, for the configuration of Fig. 1 and for one where the DT is replaced by an equivalently massed Xe target; i.e., a Xe-on-Xe pinch. The $70 \mathrm{~kJ}$ energy yield for the

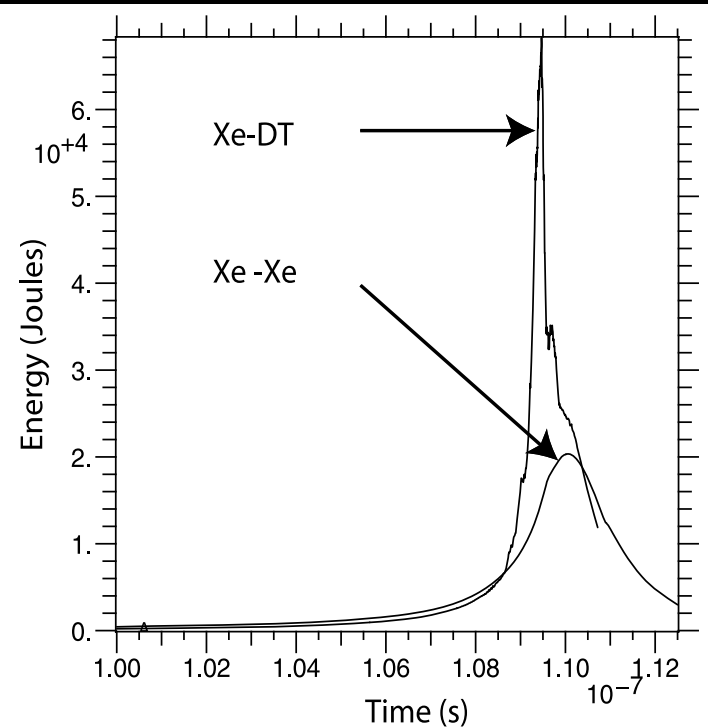

Fig. 9 Radiation energy versus time for Xe on Xe, and DT targets

Xe-on-DT pinch is approximately 3.5 times greater and the $(0.7 \mathrm{~ns}) 100 \mathrm{TW}$ power pulse is approximately 7.5 times more powerful, than for the Xe-on-Xe pinch. Nevertheless, the Xe-on-Xe pinch produces an impressive $20 \mathrm{~kJ}$ of total radiated energy and $13 \mathrm{TW}$ of power; smaller than what is typically produced in a larger-initial radius, Argon gas puff (Thornhill et al. 2007), or alternately, a Tungsten wire-array, implosion on the Z Facility (Douglas et al. 2000).

\section{Summary}

We have simulated the implosion of a Staged Z-pinch for a $100 \mathrm{~ns}, 2 \mathrm{MJ}$ facility, using the MACH2 code. The Staged Zpinch is a specific configuration consisting of a heavy, highatomic number plasma liner (Xe) imploding onto a light, low atomic number plasma target (DT). Simulations suggest that this configuration may be useful for accumulating highenergy-density in a Z-pinch plasma. The predictions are for a fusion-energy yield that is several times greater than the stored-electrical energy.

Acknowledgements Supported by the U.S. DoE. Special thanks to R.E. Peterkin and J.H. Degnan for facilitating access to the MACH2 code.

Open Access This article is distributed under the terms of the Creative Commons Attribution Noncommercial License which permits any noncommercial use, distribution, and reproduction in any medium, provided the original author(s) and source are credited.

\section{References}

Chandrasekar, S.: Hydrodynamic and Hydromagnetic Instability. Dover, New York (1981) 
Douglas, M.R., Deeney, C., Spielman, R.B., Coverdale, C.A., Roderick, N.F., Peterson, D.L.: Phys. Plasmas 7(5), 1935 (2000)

Ney, P., Rahman, H.U., Rostoker, N., Wessel, F.J.: Phys. Plasmas 8, $616(2001)$

Peterkin, R.E., Frese, M.H., Sovinec, C.R.: J. Comput. Phys. 140(1), 148 (1998). ISBN 0021-9991

Rahman, H.U., Wessel, F.J., Rostoker, N.: Phys. Rev. Lett. 74, 714 (1995)

Rahman, H.U., Ney, P., Van Drie, A., Rostoker, N., Wessel, F.J.: Phys. Plasmas 11, 5595 (2004)
Rostoker, N., Tahsiri, H.: In: Peierls, R. (ed.) Rayleigh-Taylor Instability for Impulsively Accelerated Shells; A Perspective of Physics. Gordon and Breech, New York (1978)

Teller, E.: Fusion. Academic Press, New York (1981)

Thornhill, J.W., Chong, Y.K., Apruzese, J.P., Davis, J., Clark, R.W., Giuliani Jr., J.L., Terry, R.E., Velikovich, A.L., Commisso, R.J., Whitney, K.G., Frese, M.H., Frese, S.D., Levine, J.S., Qi, N., Sze, H., Failor, B.H., Banister, J.W., Coleman, P.L., Coverdale, C.A., Jones, B., Deeney, C.: Phys. Plasmas 14, 063301 (2007) 